\title{
Probing weak forces in granular media through nonlinear dynamic dilatancy: clapping contacts and polarization anisotropy
}

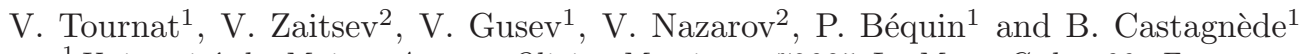 \\ ${ }^{1}$ Université du Maine, Avenue Olivier Messiaen, 72085 Le Mans Cedex 09, France \\ ${ }^{2}$ Institute of Applied Physics, 46 Uljanova Street, Nizhny Novgorod, 603950, Russia
}

(Dated: December 30, 2003)

\begin{abstract}
Rectification (demodulation) of high frequency shear acoustic bursts is applied to probe the distribution of contact forces in 3D granular media. Symmetry principles allow for rectification of the shear waves only with their conversion into longitudinal mode. The rectification is due to nonlinear dynamic dilatancy, which is found to follow a quadratic or Hertzian power law in the shear wave amplitude. Evidence is given that a significant portion of weak contact forces is localized below $10^{-2}$ of the mean force - a range previously been inaccessible by experiment. Strong anisotropy of nonlinearity for shear waves with different polarization is observed.
\end{abstract}

PACS numbers: 62.65.+k, 43.25.+y, 91.60.Lj, 62.20.Mk

Introduction. By manifesting properties of unusual solids, unusual fluids or unusual gases under certain conditions, granular media have become the subject of increasing interest to a wide audience of physical scientists [1]. For prediction of the macroscopic mechanical behavior of granular packing the knowledge of the inter-particle force distribution is essential. There is a consensus between theory and experiment concerning the abrupt exponential decay in the probability $P(f)$ of finding contacts that carry forces $f$ larger than the average force $f_{0}$ [1-7]. However, there is no consensus on the distribution of forces for those weaker than the average: there are predictions for both decreasing [4] and increasing [5-7] $P(f)$ for $f<f_{0}$. The existing experimental methods, which include the carbon paper method $[1,2,4]$, the use of the balance to measure normal forces at the bottom of the packings [3], or visualization methods [4], have been lacking so far in their range of sensitivity to delineate between theories concerning the distribution of very weak forces $\left(f \ll f_{0}\right)$. In reality, all these methods probe effects that increase with inter-particle force, and, as a result, give measurements in which response of heavily stressed contacts dominates.

Under these circumstances it is highly desirable to develop an experimental method in which the signal from the weak contacts is higher than from the strong contacts. To satisfy this requirement it is proposed in this Letter to use nonlinear acoustics methods, which are known to be selectively sensitive, in contradiction to simple intuition, to the weakest mechanical structural features of the material [8]. In contrast with earlier experimental methods [1-4], the nonlinear acoustic method described here provides information on $P(f)$ in the bulk, but not at the surface, of three-dimensional granular structures in the previously inaccessible range below a few percents of $f_{0}$. The experimental method is based on propagation of bursts of high-frequency (HF) acoustic waves to produce rectification (demodulation) which is recorded to determine $P(f)$. In nonlinear acoustics [9, 10], particularly in underwater acoustics [9], the device based on this principle is called the nonlinear parametric antenna (NPA).
In the research described here the shear $(\mathrm{S})$ wave based NPA has been used for the first time. The excitation of the low-frequency $\mathrm{S}$-wave due to rectification of $\mathrm{HF}$ $\mathrm{S}$-waves is known to be forbidden by the symmetry principle [11]. The operation of the shear NPA is possible due to dilatancy $[12,13]$, the tendency of a granular material to expand upon shearing providing nonlinear conversion of S- into longitudinal (L) waves. Indeed, the dilatancy occurs (is positive) for any sign of shear action since its dependence on S-strain is an even function, which means that this phenomenon is nonlinear in its origin, even in the case of a linear proportionality between the magnitudes of the applied shear and the induced dilatancy. The observation of S-wave rectification with conversion into L-wave can be used for determining the amplitude law of the dynamic dilatancy. The choice of elastic S-waves was additionally motivated by the expectation that the nonlinearity of the granular packing has anisotropy and this anisotropy might be probed by rotating the polarization of the shear wave.

Preliminary arguments. Here we present instructive arguments elucidating why nonlinear acoustic effects are preferentially sensitive to presence of the weakest contacts, which hardly manifest themselves in linear sound propagation. The Hertz nonlinearity of individual contacts [14] yields, in the simplest case of equal contact loading, the following relationship between macroscopic stress $\sigma$ and strain $\varepsilon$ of the material:

$$
\sigma=b n \varepsilon^{3 / 2} H(\varepsilon) \text {. }
$$

Here the factor $b$ depends on elastic moduli of the individual grains, $n$ is the average number of the contacts per grain and the Heaviside function $H(\varepsilon)$ indicates that only compressed $(\sigma, \varepsilon>0)$ contacts contribute to the stress in the material. In real granular materials there are differently loaded contacts [1-7] that contribute to the resultant $\sigma(\varepsilon)$. To illustrate the role of the different contacts in linear and nonlinear phenomena let us suppose that the granular material contains only two fractions of contacts which are differently strained. Separating explicitly the static $\left(\sigma_{0}, \varepsilon_{0}\right)$ and oscillatory $(\widetilde{\sigma}, \widetilde{\varepsilon})$ parts in the total 
macroscopic stress and strain, and adding the contributions to total stress from both fractions we get with the use of Eq.(1):

$\sigma_{0}+\widetilde{\sigma}=b n_{1}\left(\varepsilon_{0}+\widetilde{\varepsilon}\right)^{3 / 2} H\left(\varepsilon_{0}+\widetilde{\varepsilon}\right)+b n_{2}\left(\mu \varepsilon_{0}+\widetilde{\varepsilon}\right)^{3 / 2} H\left(\mu \varepsilon_{0}+\widetilde{\varepsilon}\right)$

Here $n_{1}$ and $n_{2}$ are the mean numbers of contacts per grain of two considered fractions. The dimensionless parameter $\mu$ in Eq.(2) takes into account that the static prestrain of the two fractions is different while the dynamic strain is the same. The reason for this may be understood from the evaluation of the strain in the straight vertical chains of beads presented for illustration in Fig.1. If the height $h$ of the chains oscillates near its average value $h=$ $h_{0}+\widetilde{h}$ then the strain in the chain composed of $N$ beads of a diameter $d$ each, is equal to $\varepsilon=\left(N d-h_{0}-\widetilde{h}\right) / N d$. Let the bead number $N_{0}=h_{0} / d$ correspond to zero strain in the absence of acoustic loading. Counting the number of beads in i-th column relative to this neutral level $\left(N_{i}=N_{0}+\Delta N_{i}, i=1,2\right)$ and taking into account that $\Delta N_{i} / N_{0} \ll 1$ and $|\widetilde{h}| / h_{0} \ll 1$ the strain in the column can be approximated by $\varepsilon_{i} \simeq \Delta N_{i} / N_{0}+\widetilde{h} / h_{0}$. Consequently the dynamic strain component $\widetilde{h} / h_{0}=\widetilde{\varepsilon}$ is the same for grains belonging to different chains because they have the same static height. In contrast, the static strain $\varepsilon_{0}^{(i)} \equiv \Delta N_{i} / N_{0}$ for the two columns presented in Fig. 1 is different since they contain a different number of grains $\left(\Delta N_{1} \neq \Delta N_{2}\right)$. Note that the difference in $\varepsilon_{0}^{(i)}$ for grains in these columns can be very strong even for $\Delta N_{i} / N_{0} \ll 1$. Clearly the above model is a quasi-1D version of what is expected when force chains shield a part of the grains ("spectators") from being strained. However, even in 3D packings (with essential tortuosity of force chains) the dynamic strain in the first approximation is the same at all the contacts.

In Eq.(2) it is assumed that the second fraction is weakly loaded in comparison with the first one $(\mu \ll 1)$. In the considered geometry the meaning of $\mu$ becomes clear: $\mu=\Delta N_{2} / \Delta N_{1} \ll 1$. Then under the assumption $n_{1} \sim n_{2}$ it follows that it is the first fraction that predominantly carries the static loading and $\varepsilon_{0}^{(1)}=\Delta N_{1} / N_{0}$ approximates the macroscopic strain $\varepsilon_{0}$. Summation over a unit area of the elastic forces from all chains with different preloading yields the macroscopic stress in Eq.(2).

For initially compressed contacts with $\mu>0$ and $|\widetilde{\varepsilon}| \ll \mu \varepsilon_{0}$, Eq.(2) can be expanded into series in powers $\widetilde{\varepsilon}^{m}$ with expansion coefficients $d^{m} \widetilde{\sigma}\left(\varepsilon_{0}\right) / d \widetilde{\varepsilon}^{m}$ characterizing the linear and nonlinear elastic moduli $M_{m}$ of the material:

$M_{m} \sim \frac{d^{m} \tilde{\sigma}\left(\varepsilon_{0}\right)}{d \widetilde{\varepsilon}^{m}}=\frac{3}{2} . .\left(\frac{3}{2}-m\right) b n_{1}\left(1+\frac{n_{2}}{n_{1}} \mu^{3 / 2-m}\right) \varepsilon_{0}^{3 / 2-m}$.

Eq. (3) indicates that to the linear acoustic signal (term with $m=1$ ) the contribution of the weak contacts is proportional to $\mu^{1 / 2} \ll 1$, and which may be negligible for $n_{1} \sim n_{2}$. In contrast to this, to the nonlinearityinduced signals (terms with $m \geq 2$ ) the contribution (a)

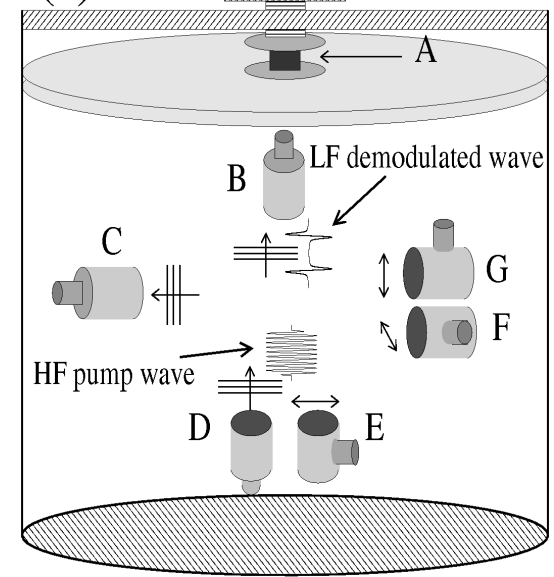

(b)

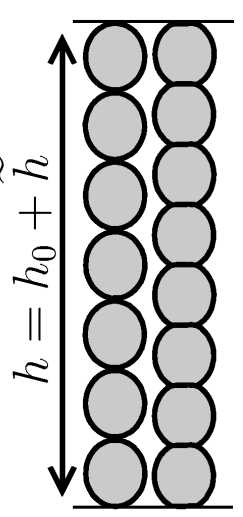

FIG. 1: (a) Diagram of the experiment. A is the force cell; B,C,D are longitudinal- and E,F,G are shear- transducers. Propagation lengths are $15-20 \mathrm{~cm}$. (b) Two grain chains with essentially different static strains are sketched.

of the weak fraction is proportional to $\mu^{3 / 2-m} \gg 1$, which dominates at sufficiently small static pre-strains $\mu \leq 0.1-0.01$. Such strains correspond to even smaller forces $f / f_{0} \leq 0.03-0.001$ which are far beyond the data region $f / f_{0} \geq 0.1$ commonly accessible by other methods $[1-4]$.

In accordance with Eq.(3), for a small enough primary acoustic wave amplitude $\varepsilon_{p} \equiv|\widetilde{\varepsilon}|<|\mu| \varepsilon_{0}$ (when the power-series expansion of Eq.(2) is valid) the rectified signal should be quadratic in $\varepsilon_{p}$ : $\langle\widetilde{\sigma}\rangle \sim M_{2} \varepsilon_{p}^{2}$. For stronger amplitude $\mu \varepsilon_{0}<\varepsilon_{p}<\varepsilon_{0}$, the dominating in the nonlinearity second term in Eq.(2) (related to the weak contacts) should be averaged as $\left\langle\left(\mu \varepsilon_{0}+\widetilde{\varepsilon}\right)^{3 / 2} H\left(\mu \varepsilon_{0}+\widetilde{\varepsilon}\right)\right\rangle \simeq$ $\left\langle(\widetilde{\varepsilon})^{3 / 2} H(\widetilde{\varepsilon})\right\rangle$ producing a rectified signal $\langle\sigma\rangle \sim \varepsilon_{p}^{3 / 2}$. Thus ocurrence of the transition $2 \rightarrow 3 / 2$ in the amplitude behavior of the demodulated signal at certain $\varepsilon_{p}$ should indicate the existence of the contacts with $\mu \sim \varepsilon_{p} / \varepsilon_{0}$.

Experimental setup. We observed the features of the demodulation of intensive L- and S - elastic waves ("pump") in glass beads $2 \pm 0.1 \mathrm{~mm}$ in diameter packed in a plastic cylindrical container, $40 \mathrm{~cm}$ in diameter and $50 \mathrm{~cm}$ in height (Fig.1). The vertical loading via a rigid plastic cover was controlled by a force cell (static stressand strain-ranges were $10-50 \mathrm{kPa}$ and $(1-5) \times 10^{-4}$, respectively). L- and S- transducers (respectively $4 \mathrm{~cm}$ and $3.5 \mathrm{~cm}$ in diameter) produced the pump bursts with carrier frequency of $30-80 \mathrm{kHz}$. The same type L-transducers were used for reception. Orientations and polarizations of the transducers are shown in Fig.1.

Evidence for weak forces localization. The radiated HF modulated pump L- and S-waves were demodulated as a result of contact nonlinearity of the granular medium. The HF pump decayed within a few $\mathrm{cm}$ distance, so that only the demodulated LF-signal of $\sim 4-6 \mathrm{kHz}$ char- 


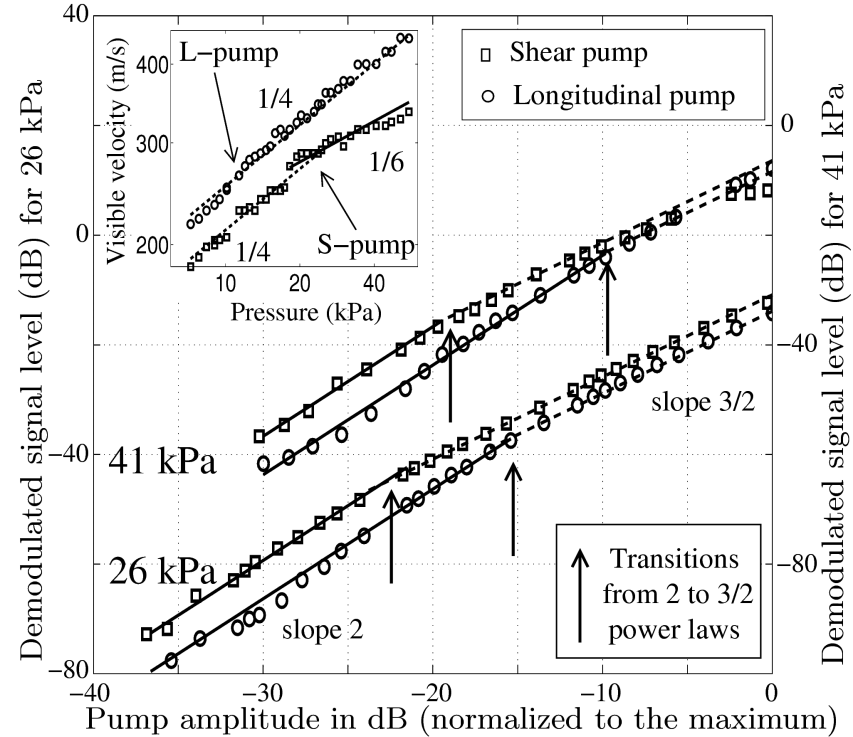

FIG. 2: Demodulated signal amplitude versus pump amplitude (vertical propagation). Inset: pressure-dependence of visible (via the time delay) velocities of the demodulated pulses (with slope $1 / 4$ higher than $1 / 6$ expected for equally loaded contacts) indicates gradual activation of weak contacts.

acteristic frequency was received. In Fig.2 the observed dependencies of the LF-signal amplitude on the L- and Spump amplitude are shown. The main feature for both L- and S- pump in Fig.2 is the initial quadratic increase in the demodulated signal amplitude with a rather clear transition to the 3/2 law corresponding to the Hertz clapping nonlinearity. Importantly, this transition occurs at an oscillating pump strain $\varepsilon_{p} 15-20 \mathrm{~dB}$ lower than the mean static strain $\varepsilon_{0}$, which, as elucidated above, is a signature of weak clapping contacts indicating strong localization of the contact-force distribution $P(f)$ below a few percents of the mean force. For Hertzian contacts where $f / f_{0}$ is proportional to $\mu^{3 / 2}$, the contactforce distribution $P(f)$ can be expressed as a contactstrain distribution $n(\mu)$, or, vice versa, through the relation $P(f) d f \propto n(\mu) d \mu$. It is often argued $[2,3]$ that the force distribution below the mean value $f_{0}$ has a plateau $P(f)=$ const., or it is at least rather flat on a logarithmic scale $[5,6]$. However, we made a simple computation of the rectified signal $\langle\sigma\rangle$ using in Eq. $(2) n(\mu)=$ const. over the range $0 \leq \mu \leq 1$, this distribution being equivalent to $P(f) \sim f^{-1 / 3}$, which is rather close to theoretical prediction [5]. This computation indicates, that the demodulated signal at sinusoidal excitation remains nearly perfectly quadratic in $\varepsilon_{p}$ for the whole range of normalized pump-strain amplitude (Fig.3 lower curve), despite the clapping of weak contacts with $\mu \leq \varepsilon_{p} / \varepsilon_{0}$. Indeed, since the distribution $n(\mu)=$ const. has no fraction strongly localized at $\mu \ll 1$, the amount of clapping contacts essentially grows with an increase in $\varepsilon_{p}$. The re-

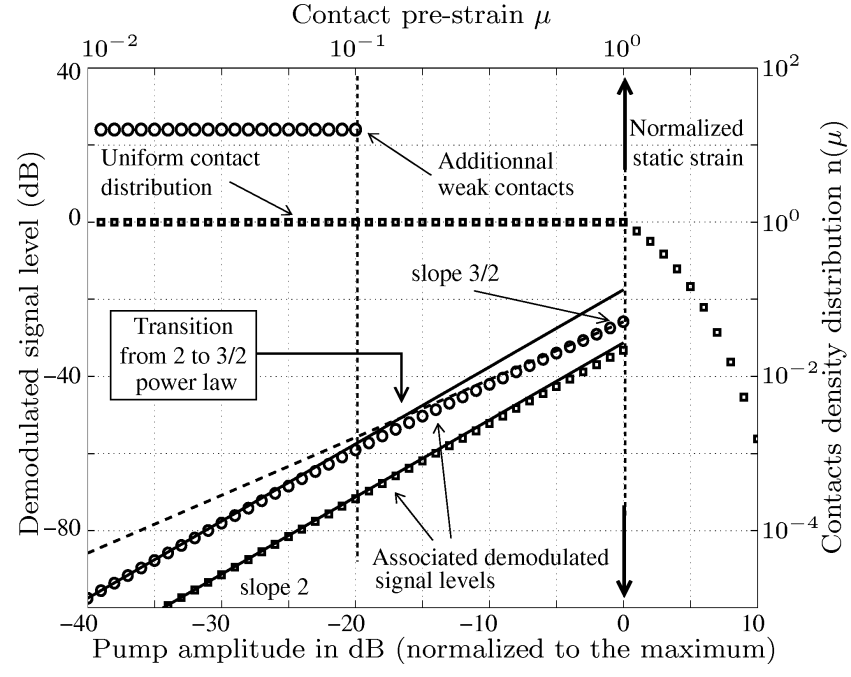

FIG. 3: Demodulated signal amplitude simulated for constant $n(\mu)$ at $0 \leq \mu \leq 1$ (squares) and in presence of localized weak fraction (circles) containing $60 \%$ of the total contact amount. The latter simulation provides clear transition $2 \rightarrow 3 / 2$ in the slope at $\varepsilon_{p} \ll \varepsilon_{0}$.

sultant demodulated signal even from clapping contacts thus grows stronger than $\varepsilon_{p}^{3 / 2}$ and remains nearly proportional to $\varepsilon_{p}^{2}$ until $\varepsilon_{p} / \varepsilon_{0}>1$, at which point practically all contacts in the material begin to clap producing $\langle\sigma\rangle \sim \varepsilon_{p}^{3 / 2}$. Therefore the occurrence of the $2 \rightarrow 3 / 2$ transition at a point much earlier than when $\varepsilon_{p} / \varepsilon_{0}>1$ clearly indicates the existence of an important fraction of contacts strongly localized below $\mu=0.1$. In the clapping regime, it is the total number of clapping contacts with $\mu \ll \varepsilon_{p} / \varepsilon_{0}$ that has important influence on the demodulated signal amplitude. Thus, to simulate the effect it is enough to add to the background uniform distribution at $0 \leq \mu \leq 1$ a weak contact fraction localized in the rectangle $0 \leq \mu \leq \mu_{0} \ll 1$ (in Fig. $3 \mu_{0}=0.1$ has been chosen). Fine details of $n(\mu)$ at $\mu \ll 1$ are difficult to reconstruct because of the integral character of its manifestation. However, it is clear that localisation should be rather strong. At the same time, to fit the experimental data in Fig.3 the boundary of the strongly localized contact-strain distribution should not be too low (otherwise, for example, $\mu_{0}=0.01$ would provide the transition $2 \rightarrow 3 / 2$ located an order of magnitude lower in $\varepsilon_{p}$ than it was in experiment).

Nonlinear dilatancy. The classical Reynolds dilatancy in the quasistatic deformation of rigid frictionless granules [12] can be qualitatively understood pure kinematically [13] as a combination of grains sliding and rotation past each other. Both the kinematics of incompressible beads [13] and the linearisation of the hypoplasticity equations [15] for granular materials predict the linear volumetric expansion $\left(\sim\left|\varepsilon_{\text {shear }}\right|\right)$ of the granular material under shear. Such an even-type piece-wise linear dependence should result in appearance of a demodulated 


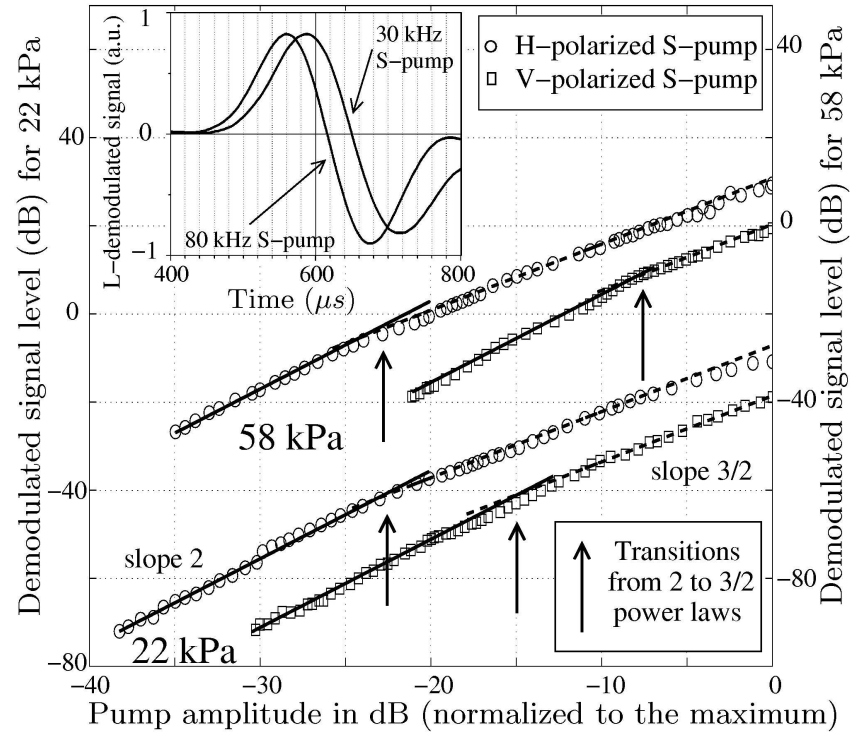

FIG. 4: Demodulated signal level versus S-pump amplitude (horizontal paths, $\mathrm{H}$ - and $\mathrm{V}$ - polarizations). Inset: demodulated-pulse profiles corresponding to the 2 nd derivative of the leading edge of long S-pump bursts.

longitudinal signal linearly proportional to the S-pump amplitude. Concerning the expected S- to L-wave conversion, the experiments with shear pump clearly indicated that the demodulated signal was indeed longitudinal judging from both polarization of the LF-pulses and their estimated velocity almost as high as L-wave velocity. The inset in Fig. 4 shows the LF-pulse shapes for two different S-pump frequencies. For lower-frequency pump (that is longer interaction length with the slower pump S-wave) the pulse acquires additional delay, which was not observed for different L-pump frequencies. However, the observed amplitude character of the S-pump demodulation indicates no signs of linear dilatancy [13, 15] in the granular material up to $\varepsilon_{p} \sim \varepsilon_{0} \sim(1-5) \times 10^{-4}$, since the demodulated L-pulses exhibit a 2 or $3 / 2$ power in the dependence on the S-pump amplitude, thus indicating that the dilatancy is essentially nonlinear. Note that quadratic dynamic dilatancy is predicted theoretically in homogeneous materials [11]. Our experimental results demonstrate for the first time an acoustic (dynamic) dilatancy following the power $3 / 2$ of the shear strain amplitude which the above arguments show to be the fingerprint of the clapping Hertzian contacts.

Probing contact anisotropy by $S$-waves. Shear waves can also be used to probe the contact anisotropy and presence of force chains oriented along the applied stress direction through the polarization dependence of the demodulation effect. As noted above, the magnitude of the contact nonlinearity is inversely proportional to the static pre-strain (see e.g. Eq.(3)); thus, in an anisotropic material different effective nonlinearity should be expected for different S-wave polarizations. Figure 4 shows amplitude dependencies of the demodulated signals from identical S-pump sources directed horizontally, but having orthogonal vertical $(\mathrm{V})$ and horizontal $(\mathrm{H})$ polarizations. The plots indicate that, first, H-polarized pump produced $10 \mathrm{~dB}$ higher-amplitude signals than V-polarized pump; second, transition to clapping $(2 \rightarrow 3 / 2)$ occured $7-12$ $\mathrm{dB}$ lower in amplitude for H-polarized pump than for $\mathrm{V}$ polarized pump. Both features indicate an effective nonlinear parameter several times higher for the $\mathrm{H}$-polarized wave than for $\mathrm{V}$-polarized wave, which means that the horizontal contacts are indeed more weakly loaded than vertical ones by rougly order of magnitude. For a HF shear-pump having circular polarization rotating with frequency $\Omega$, this anisotropic dilatancy may result in a demodulated L-wave at even harmonics $2 k \Omega, k=1,2 \ldots$

Conclusions. The results obtained here confirm that nonlinear acoustic effects can selectively probe weak contact portion of the force distribution in granular media despite a rather high background of strong force contacts. In order to explain the signal magnitude and the clear transition $2 \rightarrow 3 / 2$ in the amplitude dependence of the demodulated wave (for the first time found in a granular material) it is necessary to assume large fraction of weak contacts, over $60-70 \%$ of the total. Moreover, this transition indicates that the distribution of weak contacts contains a significant fraction strongly localized near zero force. For irregular grain-shapes, like in dry sand, the localization is even stronger, since the quadratic behavior of the power law could not be observed at all [10]. The localization extracted from our experiments is strong in the sense that it is inconsistent with a smooth power law of the form $P(f) \propto f^{-\alpha}\left(f<f_{0}\right)$ theoretically predicted both for 2D monodisperse granular systems with friction $(\alpha=0.5)[6]$ and $2 \mathrm{D}$ polydisperse frictionless systems $(\alpha=0.3)$ [5]. The experimental results obtained here agree qualitatively with recent $3 \mathrm{D}$ molecular dynamics simulation [7] of monodisperse unloaded packings with friction indicating an abrupt upturn in $P(f)$ at very small values of $f\left(f \leq 0.1 f_{0}\right)$, and should stimulate further theoretical modeling.

We believe that the extension of the research might be related with application of the NPA to the evaluation of the slow dynamics (in particular, of such irreversible processes as compaction and contacts heating [8]) and observation of polarization-sensitive nonlinear effects. The optimization of a compact highly-directive parametric emitter and combination of L- and S- pump waves for the diagnostics of granular piles might be suggested as a possible practical application.

The study was partially supported by RFBR (grants 02-02-08021-inno and 02-02-16237). 
[1] H. M. Jaeger, S. R. Nagel, R. P. Behringer, Rev. Mod. Phys. 68, 1259 (1996). M. E. Cates, J.P. Wittmer, J.-P. Bouchaud, P. Claudin, Phys. Rev. Lett. 81, 1841 (1998).

[2] D. L. Blair et al., Phys. Rev. E 63, 041304 (2001).

[3] G. Lovoll, K. J. Maloy, E. G. Flekkoy, Phys. Rev. E 60, 5872 (1999).

[4] C.-h. Liu, et al., Science 269, 513 (1995).

[5] F. Radjai et al., Phys. Rev. Let. 77, 274 (1996).

6] S. Luding, Phys. Rev. E 55, 4720 (1997).

[7] L. E. Silbert, G. S. Grest, J. W. Landry, Phys. Rev. E 66, 061303 (2002).

[8] I. Yu. Belyaeva, V. Yu. Zaitsev, Acoust. Phys. 43, 594 (1997); V. Zaitsev, V. Gusev, B. Castagnède, Phys. Rev. Lett. 89, 105502 (2002).
[9] B.K. Novikov, O.V. Rudenko, V.I. Timochenko, Nonlinear Underwater Acoustics, (ASA, New-York, 1987).

[10] V.Y. Zaitsev, A.B. Kolpakov and V.E. Nazarov, Acoust. Phys. 45, (Pt.I) 235, (Pt.II) 347 (1999).

[11] L. K. Zarembo, V. A. Krasilnikov, Sov. Phys. Uspekhi 13, 778 (1971)

[12] O. Reynolds, Philos. Mag. 20, 469 (1885).

[13] J. D. Goddard, A. K. Didwania, Q. Jl. Mech. Appl. Math. 51, 15 (1998).

[14] K.L. Johnson, Contact Mechanics, (Cambridge University Press, Cambridge, 1985).

[15] D. Kolymbas, Int. J. Numer. Anal. Meth. Geomech. 19, 415 (1995). 